\title{
Herpesvirus Vectors for Therapy of Brain Tumors
}

\author{
Kevin A. Cassady and Jacqueline Nuss Parker* \\ Department of Pediatrics, Division of Infectious Diseases, University of Alabama at Birmingham, Birmingham, AL \\ 35294-0011, USA
}

\begin{abstract}
Genetically modified, conditionally-replicating Herpes Simplex Virus Type 1 (HSV-1) vectors for the treatment of malignant glioma have provided encouraging results in the handful of Phase I and Phase II clinical trials conducted to date. In recent years, a number of new strategies have been developed to improve anti-tumor activity of these attenuated vectors, through either introduction of foreign gene inserts to enhance tumor killing through a variety of mechanisms, or through combination with existing treatment regimens, including radiation and/or chemotherapeutics. Another promising new approach has been the engineering of novel oncolytic HSV vectors that retain wildtype replication, but are targeted to tumor cells through a variety of mechanisms. This review summarizes the latest advances in herpesvirus-mediated oncolytic therapies from both preclinical results and clinical trials with oncolytic HSV vectors in patients, and their implication for design of future trials.
\end{abstract}

Keywords: Oncolytic HSV therapy, brain tumor, G207, HSV1716, M032, chimeric HSV, C134, R5141, rQNestin34.5, phase I trial, glioma, $\gamma_{1}(34.5)$.

\section{INTRODUCTION}

Primary and metastatic tumors arising in the brain remain a therapeutic challenge. For malignant glioma, the most frequently occurring primary brain tumor, novel treatment regimens have extended survival only by a few months. Nearly three decades ago, advancements in molecular biology, combined with research into viral-based experimental therapies for a number of malignancies including glioma, generated significant interest in the application of genetically engineered, conditionallyreplicating herpes simplex viruses (HSV-1) vectors for use as anti-tumor agents. A number of recent reviews of the history of oncolytic viral vectors, including those derived from HSV-1, and their application for therapy of CNS malignancies have summarized advances in oncolytic viral therapy over the past several years [1-3]. The emphasis of the current review is to provide an overview of the latest approaches and technologies utilizing both attenuated, conditionally replicating vectors, as well as HSV vectors that replicate with wildtype efficiency but in a tumor-specific fashion. These include:

i) Introduction of foreign gene inserts that enhance vector-mediated oncolysis through a variety of mechanisms;

ii) Use of chimeric HSV/human cytomegalovirus (HCMV) vectors in which introduction of the HCMV IRS1 or TRS1 genes into HSV backbone restores the wildtype protein synthesis phenotype without concomitant restoration of neurovirulence;

\footnotetext{
*Address correspondence to this author at the Department of Pediatrics, Division of Infectious Diseases, University of Alabama at Birmingham, Birmingham, AL 35294-0011, USA; Tel: 205-975-6549; Fax: 205-9967881; E-mail: JParker@peds.uab.edu
}

iii) Combining oncolytic HSV therapy with standard of care therapies, including radiation and chemotherapeutics;

iv) Development of wildtype, nonattenuated HSV vectors that are retargeted to tumor-specific receptors;

v) Development of attenuated HSV vectors in which the HSV-1 neurovirulence gene, gamma-1 $34.5\left(\gamma_{1} 34.5\right)$, discussed in more detail below, is expressed from a tumor-specific promoter (nestin 1); and

vi) Improved methods for delivery of oncolytic viral vectors, including convection-enhanced delivery.

Each of these therapies is discussed in more detail in subsequent subsections, and summarized schematically in Fig. (1). Finally, an update of latest clinical trial results, both recently completed and pending, is provided at the end, and summarized in Table $\mathbf{1}$.

\section{ATTENUATING MUTATIONS OF ONCOLYTIC HERPES SIMPLEXVIRUS VECTORS}

dlsptk mutants In the mid to late 1970s, an HSV-1 mutants that lacked a functional thymidine kinase gene were shown to be incapable of virus replication in both nondividing cells and in the mammalian nervous system [4]; subsequently, a tk deletion virus, named $d l s p t k$, was shown to be capable of establishing, but not reactivating from, latency [5]. This HSV mutant was shown in 1991 by Martuza and colleagues to effectively kill glioma cell lines in vitro and in subcutaneous tumors, and prolonged survival of mice bearing intracranial tumors established from the same glioma cell line [6]. The $t k$ gene deletion rendering this mutant resistant to antiviral agents that target the viral thymidine kinase prevented advancement of this virus to clinical trials in patients. Nonetheless, these pioneering studies demonstrated that HSV-1 derived vectors with 


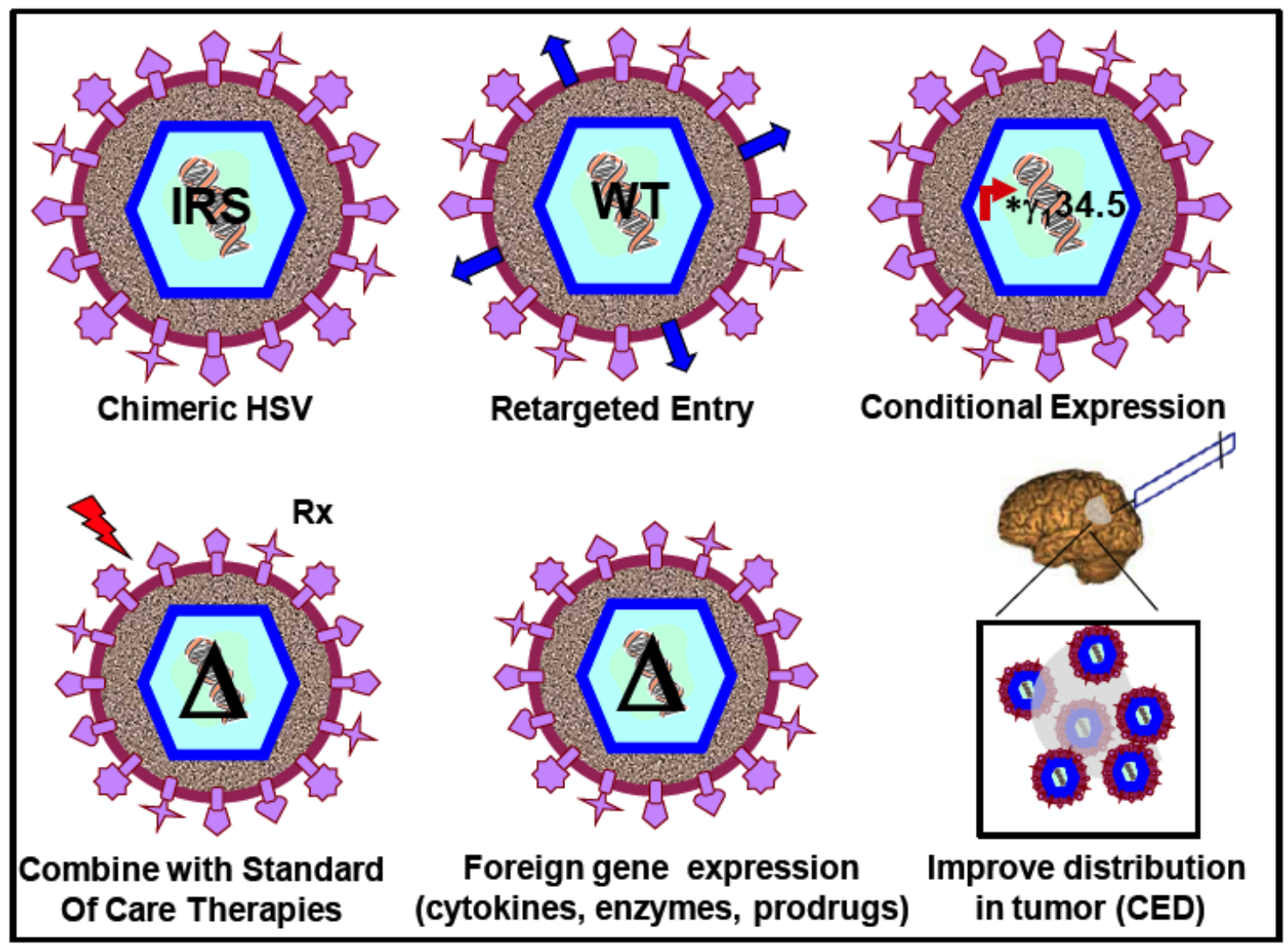

Fig. (1). Strategies to improve oHSV therapy of malignant glioma. Each of the different strategies discussed in detail in the text are shown schematically. IRS $=\mathrm{C} 134$ chimeric HSV expressing IRS gene from HCMV; WT= retargeted viruses that have wildtype ICP34.5 but can only infect cells with upregulated tumor-specific receptors; ${ }^{*} \gamma_{1} 34.5=$ ICP34.5 expressed from tumor-specific promoters; $\Delta=\gamma_{1} 34.5$ deleted viruses.

attenuating mutations could be used for specific treatment of rapidly growing tumors in the brain.

Ribonucleotide reductase mutants. The "proof of concept" studies from the dlsptk mutants emphasized the need to develop viral vectors avirulent in normal brain but capable of replication in actively dividing tumor populations. The HSV-1 mutant hrR3 was constructed in which the bacterial lacZ gene was introduced within the UL39 gene encoding the viral ribonucleotide reductase (infected cell protein 6, or ICP6) [7]. The native thymidine kinase was retained in this construct; as such this mutant retained sensitivity to ganciclovir. Lack of a functional ICP6 protein prevented the virus from replicating in nondividing cells. However, in actively dividing cells, the virus was still able to replicate, though progeny virus recovery was reduced as compared to wildtype HSV. Additionally, anti-tumor activity of rat brain tumors following treatment with hrR3 is potentiated by ganciclovir treatment [8]. The HSV-1 vector G207 used in three Phase I clinical trials in the United States also contains a lacZ insertion in the $\mathrm{U}_{\mathrm{L}} 39$ gene similar to hrR3, in addition to deletions of both copies of the $\gamma_{1} 34.5$ gene, described in the next section.

భ-1 34.5 deleted HSV mutants. In 1990, Chou and Roizman demonstrated that the neurovirulence function of HSV-1 mapped to the diploid gene $\gamma_{1} 34.5$, located in the inverted repeat sequences flanking the Unique Long $\left(\mathrm{U}_{\mathrm{L}}\right)$ segment of the viral genome [9]. In later studies, the normal function of ICP34.5, the protein product encoded by the $\gamma_{1} 34.5$ gene, was shown to preclude the shutoff of host protein synthesis by recruitment of the host protein phosphatase-1a, and subsequent dephosphorylation of the eukaryotic translation initiation factor alpha (eIF-2 $\alpha)$ [10]. Normally, following infection with wild type HSV-1, production of double stranded RNA triggers an intracellular stress response that causes the protein kinase R (PKR) to phosphorylate eIF- $2 \alpha$, which mediates protein synthesis shutoff. In normal non-mitotic cells, deletion or disruption of both copies of the $\gamma_{1} 34.5$ gene severely limit virus replication due to the host PKR-mediated shutoff of host protein synthesis. However replication of $\gamma_{1} 34.5$-deleted HSV can occur in tumor cells that possess complementing mutations. Examples include ras overexpression [11], and alterations in PKR and other cell signaling pathways [12]. Of note, deletion of the $\gamma_{1} 34.5$ gene also impacts synthesis of the latency-activated transcripts (LATs), encoded on complementary antisense DNA strands. To date, both oncolytic HSV vectors tested in patient clinical trials are based on the $\gamma_{1} 34.5$-deletion platform (for recent reviews, see $[1,2])$.

\section{OHSV VECTORS FOR DELIVERY OF FOREIGN GENE-MEDIATED THERAPIES}

Oncolytic virus therapy using HSV-1 vectors have a number of advantages, one being its capability for introduction of large transgenes for combining oHSV 
Table 1. Past, Present and Pending oHSV Trials in Patients with Malignant Glioma

\begin{tabular}{|c|c|c|c|c|c|}
\hline Virus & Parent Strain & Tumor-Targeting Mutation(s) & Trial Description & Trial Status & References \\
\hline \multirow{4}{*}{ HSV1716 } & \multirow{4}{*}{17} & \multirow{4}{*}{ Both $\gamma_{1} 34.5$ gene copies deleted } & Phase 1 dose escalation, safety & closed & {$[21]$} \\
\hline & & & $\begin{array}{l}\text { Phase } 1 \mathrm{~b} \text {, single dose, assess } \\
\text { intratumoral virus replication }\end{array}$ & closed & {$[37]$} \\
\hline & & & $\begin{array}{l}\text { Phase Ib, safety of resection } \\
\text { cavity administration }\end{array}$ & closed & {$[38]$} \\
\hline & & & Phase III & ongoing & nexxusscotland.com website \\
\hline \multirow{3}{*}{ G207 } & \multirow{3}{*}{$\mathrm{F}$} & \multirow{3}{*}{$\begin{array}{l}\text { Both } \gamma_{1} 34.5 \text { gene copies deleted, } \\
\text { lacZ gene inserted into } U_{L} 39 \text { gene }\end{array}$} & Phase 1 dose escalation, safety & closed & {$[20]$} \\
\hline & & & $\begin{array}{l}\text { Phase } \mathrm{Ib} \text {, two dose regimen, one } \\
\text { intratumor, one into resection } \\
\text { cavity }\end{array}$ & closed & [17] \\
\hline & & & Phase Ib & closed & Markert et al., submitted \\
\hline M032 & $\begin{array}{c}\text { F/ } \\
\text { M002 }\end{array}$ & Both $\gamma_{1} 34.5$ gene copies deleted & $\begin{array}{l}\text { Phase I, safety and dose } \\
\text { escalation }\end{array}$ & pending & [15] \\
\hline $\mathrm{C} 134$ & $\begin{array}{c}\mathrm{F} / \\
\mathrm{C} 101\end{array}$ & $\begin{array}{l}\text { Both } \gamma_{1} 34.5 \text { gene copies deleted, } \\
\text { HCMV gene IRS } 1 \text { introduced } \\
\text { within } \mathrm{U}_{\mathrm{L}} 3 / \mathrm{U}_{\mathrm{L}} 4 \text { intergenic region }\end{array}$ & $\begin{array}{l}\text { Phase I safety and dose } \\
\text { escalation }\end{array}$ & $\begin{array}{l}\text { planning } \\
\text { stages }\end{array}$ & [16] \\
\hline
\end{tabular}

therapy with expression of foreign genes that complement the anti-tumor activity of the vector. There are currently a number of different classes of transgenes being introduced into oHSV vectors that are being evaluated as novel therapies for glioma gene therapy. These include the introduction of genes encoding pro-drug activating enzymes, tumor suppressor genes, immune modulating genes, and genes encoding proteins that inhibit tumor angiogenesis [13, $14]$ and references therein. A number of studies by our group have demonstrated the antitumor efficacy of an IL-12 expressing oHSV, M002, in both syngeneic murine brain tumor models and human glioma xenograft tumor models [15]. M002, like G207, is derived from the HSV-1 (F) strain with deletions in both $\gamma_{1} 34.5$ genes. The other notable difference between M002 and G207 is that in M002, the $\mathrm{U}_{\mathrm{L}} 39$ gene remains intact. A bicistronic expression cassette encoding interleukin-12 p40 and p35 subunits from either murine (M002) or human (M032) origin, and separated by an internal ribosome entry sequence (IRES), were introduced into both $\gamma_{1} 34.5$ deleted sites. Production of a cGMP lot of M032 for Phase I clinical trials through the NCI Biopharmaceutical Development Program (BDP) has recently been completed, and submission of an IND application for approval by the United States Food and Drug Administration (FDA) is planned for summer 2010.

\section{CHIMERIC HSV/HCMV}

Preclinical and clinical evidence indicates that tumor cells can escape $\Delta \gamma_{1} 34.5$ first generation oncolytic HSV treatment $[16,17]$. One hypothesis is that these mutants are unable to maintain prolonged replication in the tumor, and therefore their ability to spread throughout the tumor mass is compromised by the innate antiviral host responses. To counteract these responses impeding $\Delta \gamma_{1} 34.5$ HSV replication in infected tumor cells, the IRS1 gene from a distantly related herpesvirus, Human Cytomegalovirus (HCMV), was introduced into a $\Delta \gamma_{1} 34.5 \mathrm{HSV}$ background. IRS-1 has been previously demonstrated to selectively restore late viral protein synthesis [18]. Additionally, since the HCMV gene was evolutionarily distant enough from HSV-1 genes, it was hypothesized that IRS-1 lacked the neurovirulence function of $\gamma_{1} 34.5$ and thus could restore late viral protein synthesis without restoring neurovirulence. Two chimeric HSVs were engineered; C130, a $\Delta \gamma_{1} 34.5 \mathrm{HSV}$ engineered to express the HCMV TRS1 gene and C134, a $\Delta \gamma_{1} 34.5 \mathrm{HSV}$ engineered to express HCMV IRS1. Results indicated that insertion of the HCMV TRS1 or TRS1 gene into a $\Delta \gamma_{1} 34.5 \mathrm{HSV}$ resulted in a virus with restored late viral protein synthesis and improved replication in malignant glioma cells both in vitro and in vivo [19]. This improved replication translated to a better anti-tumor response as well, when directly compared to tumors treated with parent $\Delta \gamma_{1} 34.5 \mathrm{HSV}$ only. Importantly, the HSV/HCMV chimeric viruses were also demonstrated to be safe following direct intracranial inoculation in mice, but with significantly different $\mathrm{LD}_{50}$ values. While the $\mathrm{LD}_{50}$ value measured for $\mathrm{C} 134$, which expressed IRS1, was identical to that of its parent $\Delta \gamma_{1} 34.5 \mathrm{HSV}$, the $\mathrm{LD}_{50}$ value of C130, encoding the TRS1 gene, was increased by nearly 100 fold as compared to C134. Despite the increased virulence of C130, both HSV/HCMV chimeric viruses have safety profiles similar to oHSV (HSV1716 and G207) that have been successfully used in Phase I clinical trials [20,21]. Due to its excellent anti-tumor efficacy and minimal neurotoxicity, C134 has been advanced for clinical development. An application was submitted, and received approval, for production of C134 using current Good Manufacturing Practices (cGMP) through the National Cancer Institute's Rapid Access to Intervention Development (RAID) Program for the translation of novel experimental therapeutics into Phase I trials in patients.

\section{STANDARD OF CARE THERAPIES IN COMBINATION WITH OHSV THERAPY}

The most recent shift in standard of care treatment paradigms for patients with newly diagnosed glioma was made following the report that combination of ionizing radiation with temozolomide (TMZ) administration resulted in significantly improved survival benefit $v s$ treatment with either therapy alone [22]. In this study, the median two year 
survival of patients treated with the combination therapy was over $26 \%$ vs $10 \%$ two year survival of patients that received radiation therapy only. It is not unreasonable to predict that combining oncolytic HSV therapies with standard of care therapies like ionizing radiation and chemotherapeutic drugs would also result in improved survival benefit $v s$ either therapy alone. A number of reports support this, and are summarized below.

$\Delta \gamma_{1} 34.5 H S V$ and ionizing radiation. In 1998, Advani and colleagues demonstrated that administration of high doses of ionizing radiation (IR) 24 and 48 hours following direct, intratumoral inoculation of R3616, a $\Delta \gamma_{1} 345 \mathrm{HSV}$, lysed glioma tumor explants much more efficiently than treatment with either therapy alone [23]. More recently, the underlying mechanism mediating enhanced viral replication was linked to upregulation of late viral promoters by the host p38 protein [24]. These results are discussed in more detail elsewhere [25].

oHSV therapy in combination with chemotherapeutic drugs. Likewise, combination of TMZ treatment with oHSV vectors was also shown to improve lysis of tumor cells resistant to destruction by either agent alone [26]. Aghi and colleagues discovered that the DNA repair mechanisms elicited following treatment with TMZ enhance intratumoral replication of oHSV and kill tumor cell lines normally resistant to either therapy alone. These data support reports of combination radiation or chemotherapy with oncolytic viral therapy in other malignancies outside the brain $[27,28]$.

\section{TUMOR-SPECIFIC RETARGETING OF OHSV}

As mentioned earlier, one of the primary limitations of the $\Delta \gamma_{1} 34.5 \mathrm{HSV}$ platform is their inability to maintain efficient replication in CNS tumors. One solution is to engineer viruses in which the $\gamma_{1} 34.5$ gene is only expressed in tumor cells, but not in adjacent normal tissue. Two of the strategies developed to enable tumor-specific ICP34.5 expression are i) to modify virus glycoproteins to enter cells via a tumor-specific receptor, while preventing interaction with normal cell surface receptors, and ii) to express ICP34.5 under the control of a tumor-specific promoter. In a series of elegant experiments, Zhou and Roizman and colleagues were able to re-target a wildtype HSV vector to the IL-13Ralpha2 receptor, which is expressed on the surface of many glioblastoma cell lines, as well as on anaplastic astrocytomas $[29,30]$. HSV mutant R5111 contains both copies of 34.5 intact, but the known heparan binding sites on glycoproteinB (gB) and $\mathrm{gC}$ were mutated. R5111 also contains a chimeric $\mathrm{gD}$ that was constructed to express IL-13. R5111 was able to infect cells following interaction of the chimeric $\mathrm{gD} / \mathrm{IL} 13$ with the IL13Ralpha2 on the tumor cell lines tested [31]. Mutations were then introduced into R5111 to construct a virus (R5141) in which binding to either HveA or nectin-1, its native receptor has been completely abolished [32]. Current challenges for receptor re-targeted viruses like R5141 include development of an appropriate and FDAapproved cell line for production of highly purified, high titer virus stocks for preclinical safety and efficacy testing in mice, and for Phase I clinical trials in patients.

The second strategy for targeted expression of ICP34.5 employs the use of tumor-specific promoters driving expression of the ICP34.5 protein. One example is the promoter for nestin-1, an intermediate filament protein shown to be upregulated in a high percentage of human glioma cell lines, but not normal human astrocytes. Studies by Kambara and colleagues demonstrated that rQNestin34.5, an HSV engineered to express ICP34.5 from the nestin-1 promoter, was able to improve survival of mice bearing intracranial tumors as compared to treatment with the parent virus (lacking both $\gamma_{1} 34.5$ gene copies and containing a disrupted $\mathrm{U}_{\mathrm{L}} 39$ gene) only [33]. In vivo neurotoxicity studies showed that rQNestin34.5 was not more neurovirulent than its $\gamma_{1} 34.5$-deleted parent.

Another example of a tumor-specific promoter driving ICP34.5 expression is the mutant HSV KeM34.5. KeM34.5 was derived from G207, but the lacZ gene within the UL39 gene has been replaced with the Musashi-1 promoter driving ICP34.5 expression [34]. Musashi 1 is a neural RNA-binding protein whose expression is upregulated in glioma cell lines. Treatment of human glioma tumors established intracranially in mice resulted in significantly improved survival of tumor bearing mice, and this effect was dose-dependent.

Thus retargeting of oHSV vectors, either through tumorspecific receptors allowing specific delivery of wildtype virus, or through tumor-specific promoter activity controlling ICP34.5, are promising strategies for glioma therapy. It remains to be seen whether they will be safe for administration in humans.

\section{CONVECTION-ENHANCED DELIVERY (CED) OF oHSV}

Another obstacle in the treatment of malignant glioma is efficient delivery of drugs or therapeutics, including oHSV, into the tumor site. The blood brain barrier (BBB) can significantly impede delivery of novel therapeutic drugs and agents to tumors inaccessible to stereotactic injection of oHSV. Even when the tumor mass is accessible, migrating tumor cells distal from the primary mass are often left untouched, due to inadequate distribution at tumor inoculation [35]. One recently described approach to improve distribution of oHSV vectors into the tumor mass is the use of convection enhanced delivery (CED). In CED, multiple catheters are placed stereotactically within the tumor mass and surrounding the tumor/resection area. Hadjipanayis et al. demonstrated the CED of a replication defective HSV vector, d106, significantly improved survival of tumor-bearing mice when the therapy was combined with IR or TMZ [36]. However there was no significant difference in median survival rates of mice treated with d106 + CED alone (without IR or TMZ). Nevertheless, CED using any of the oHSV vectors described earlier in this review is a promising approach to ensure adequate and widespread distribution in the tumor.

\section{ADVANCING ONCOLYTIC HSV VECTORS INTO PATIENTS WITH MALIGNANT GLIOMA: STATUS OF CLINICAL TRIALS}

A total of 5 clinical trials in patients utilizing oHSV vectors have been published to date $[17,20,21,37,38]$. These trials were initiated more than a decade ago with the first-generation oHSV vectors HSV1716 (United Kingdom) and G207 (United States). Both HSV1716 (derived from wildtype strain 17) and G207 (derived from wildtype strain 
"F") have one or both of the attenuating mutations $\left(\gamma_{1} 34.5\right.$ deletion and $\mathrm{U}_{\mathrm{L}} 39$ disruption), respectively, and described earlier in this review. The details of these trials have been adequately summarized elsewhere $[1,39]$.

A Phase Ib study has just been completed in which ionizing radiation was administered following G207 in a group of 9 patients (Markert JM, personal communication) and report of the results are pending final analysis. Finally, according to their website, [http://www.nexxusscotland.com/ life_science/case_studies/organisations/crusade_laboratories], Crusade Laboratories in Glasgow, Scotland has initiated a Europe-wide Phase III clinical trial using HSV1716.

At least two other promising oHSV vectors are close to initial Phase I safety studies in patients. cGMP production of M032, mentioned earlier, is complete and, pending final sterility testing, submission of an IND application to the FDA is planned for mid to late 2010. The chimeric HSV/HCMV, C134, is also being submitted for cGMP production through the NCI BDP. Production date has not yet been set, but protocols for growing up high titer virus production lots have already been developed and the process is anticipated to proceed quickly. Table 1 summarizes the past and present oHSV clinical trials in patients, as well as second generation viruses poised to enter the clinical trial arena in the next 12-18 months.

\section{CONCLUDING REMARKS}

The field of oncolytic HSV therapy for treatment of malignant glioma continues to evolve with the advent of new therapeutics, and discovery of new tumor-specific targets to be engineered into oHSV vectors. Examples of novel targets include markers of glioma progenitor cells (GPCs), as well as tumor-specific delivery and IR-mediated or expression of proteins involved in signal transduction pathways. Gene expression profile analyses of G207-treated tumor specimens from the G207 Phase Ib study [17] should provide new insight as to correlates of responders to oHSV therapy $v s$ nonresponders. Regardless, inasmuch as the oHSV trials in patients to date have overwhelmingly demonstrated safety of direct intratumoral inoculation and surrounding adjacent tissue, advancement of $2^{\text {nd }}$ and $3^{\text {rd }}$ generation viruses into Phase I clinical trials, alone and in combination with current standard of care therapies, is eagerly anticipated.

\section{REFERENCES}

[1] Parker, JN, Bauer DF, Cody JJ, Markert JM. Oncolytic viral therapy of malignant glioma. Neurotherapeutics 2009; 6: 558-69.

[2] Grandi, P, Peruzzi P, Reinhart B, et al. Design and application of oncolytic HSV vectors for glioblastoma therapy. Expert Rev Neurother 2009; 9: 505-17.

[3] Todo, T. Oncolytic virus therapy using genetically engineered herpes simplex viruses. Front Biosci 2008; 13: 2060-4

[4] Jamieson, AT, Gentry GA, Subak-Sharpe JH. Induction of both thymidine and deoxycytidine kinase activity by herpes viruses. J Gen Virol 1974; 24: 465-80.

[5] Coen, DM, Kosz-Vnenchak M, Jacobson JG, et al. Thymidine kinase-negative herpes simplex virus mutants establish latency in mouse trigeminal ganglia but do not reactivate. Proc Natl Acad Sci USA 1989; 86: 4736-40.

[6] Martuza, RL, Malick A, Markert JM, Ruffner KL, Coen DM. Experimental therapy of human glioma by means of a genetically engineered virus mutant. Science 1991; 252: 854-6.

[7] Goldstein, DJ, Weller SK. Factor(s) present in herpes simplex virus type 1-infected cells can compensate for the loss of the large subunit of the viral ribonucleotide reductase: characterization of an ICP6 deletion mutant. Virology 1988; 166: 41-51.

[8] Boviatsis, EJ, Park JS, Sena-Esteves M, et al. Long-term survival of rats harboring brain neoplasms treated with ganciclovir and a herpes simplex virus vector that retains an intact thymidine kinase gene. Cancer Res 1994; 54: 5745-51.

[9] Chou, J, Kern ER, Whitley RJ, Roizman B. Mapping of herpes simplex virus-1 neurovirulence to gamma 134.5, a gene nonessential for growth in culture. Science 1990; 250: 1262-6.

[10] He, B, Gross M, Roizman B. The gamma134.5 protein of herpes simplex virus 1 has the structural and functional attributes of a protein phosphatase 1 regulatory subunit and is present in a high molecular weight complex with the enzyme in infected cells. J Biol Chem 1998; 273: 20737-43.

[11] Farassati, F, Yang AD, Lee PW. Oncogenes in Ras signalling pathway dictate host-cell permissiveness to herpes simplex virus 1 . Nat Cell Biol 2001; 3: 745-50.

[12] Chou, J, Roizman B. The gamma 1(34.5) gene of herpes simplex virus 1 precludes neuroblastoma cells from triggering total shutoff of protein synthesis characteristic of programed cell death in neuronal cells. Proc Natl Acad Sci USA 1992; 89: 3266-70.

[13] Asadi-Moghaddam, K, Chiocca EA. Gene- and viral-based therapies for brain tumors. Neurotherapeutics 2009; 6: 547-57.

[14] Kaur, B, Cripe TP, Chiocca EA. "Buy one get one free": armed viruses for the treatment of cancer cells and their microenvironment. Curr Gene Ther 2009; 9: 341-55.

[15] Parker, JN, Gillespie GY, Love CE, et al. Engineered herpes simplex virus expressing IL-12 in the treatment of experimental murine brain tumors. Proc Natl Acad Sci USA 2000; 97: 22082213.

[16] Shah, AC, Parker JN, Gillespie GY, et al. Enhanced antiglioma activity of chimeric HCMV/HSV-1 oncolytic viruses. Gene Ther 2007; 14: 1045-54.

[17] Markert, JM, Liechty PG, Wang W, et al. Phase Ib trial of mutant herpes simplex virus G207 inoculated pre-and post-tumor resection for recurrent GBM. Mol Ther 2009; 17: 199-207.

[18] Child, SJ, Hakki M, De Niro KL, Geballe AP. Evasion of cellular antiviral responses by human cytomegalovirus TRS1 and IRS1. J Virol 2004; 78: 197-205.

[19] Cassady, KA, Human cytomegalovirus TRS1 and IRS1 gene products block the double-stranded-RNA-activated host protein shutoff response induced by herpes simplex virus type 1 infection. J Virol 2005; 79: 8707-15

[20] Markert, JM, Medlock MD, Rabkin SD, et al. Conditionally replicating herpes simplex virus mutant, G207 for the treatment of malignant glioma: results of a phase I trial. Gene Ther 2000; 7: 867-74.

[21] Rampling, R, Cruickshank G, Papanastassiou V, et al. Toxicity evaluation of replication-competent herpes simplex virus (ICP 34.5 null mutant 1716) in patients with recurrent malignant glioma. Gene Ther 2000; 7: 859-66.

[22] Stupp, R, Mason WP, van den Bent MJ, et al. Radiotherapy plus concomitant and adjuvant temozolomide for glioblastoma. N Engl J Med 2005; 352: 987-96.

[23] Advani SJ, Sibley GS, Song PY, et al. Enhancement of replication of genetically engineered herpes simplex viruses by ionizing radiation: a new paradigm for destruction of therapeutically intractable tumors. Gene Ther 1998; 5: 160-5.

[24] Mezhir JJ, Advani SJ, Smith KD, et al. Ionizing radiation activates late herpes simplex virus 1 promoters via the p38 pathway in tumors treated with oncolytic viruses. Cancer Res 2005; 65: 947984.

[25] Advani SJ, Mezhir JJ, Roizman B, Weichselbaum RR. ReVOLT: radiation-enhanced viral oncolytic therapy. Int J Radiat Oncol Biol Phys 2006; 66: 637-46.

[26] Aghi M, Rabkin S, Martuza R. Effect of chemotherapy-induced DNA repair on oncolytic herpes simplex viral replication. J Natl Cancer Inst 2006; 98: 38-50.

[27] Jarnagin, WR, Zager JS, Hezel M, et al. Treatment of cholangiocarcinoma with oncolytic herpes simplex virus combined with external beam radiation therapy. Cancer Gene Ther 2006; 13: 326-34.

[28] Stanziale SF, Petrowsky H, Joe JK, et al. Ionizing radiation potentiates the antitumor efficacy of oncolytic herpes simplex virus G207 by upregulating ribonucleotide reductase. Surgery 2002; 132: 353-9. 
[29] Debinski W, Gibo DM, Hulet SW, Connor JR, Gillespie GY. Receptor for interleukin 13 is a marker and therapeutic target for human high-grade gliomas. Clin Cancer Res 1999; 5: 985-90.

[30] Mintz A, Gibo DM, Slagle-Webb B, Christensen ND, Debinski W, IL-13Ralpha2 is a glioma-restricted receptor for interleukin-13. Neoplasia 2002; 4: 388-99.

[31] Zhou G, Roizman B. Characterization of a recombinant herpes simplex virus 1 designed to enter cells via the IL13Ralpha2 receptor of malignant glioma cells. J Virol 2005; 79: 5272-7.

[32] Zhou G, Roizman B. Construction and properties of a herpes simplex virus 1 designed to enter cells solely via the IL-13alpha2 receptor. Proc Natl Acad Sci USA 2006; 103: 5508-13.

[33] Kambara H, Okano H, Chiocca EA, Saeki Y. An oncolytic HSV-1 mutant expressing ICP34.5 under control of a nestin promoter increases survival of animals even when symptomatic from a brain tumor. Cancer Res 2005; 65: 2832-9.

[34] Kanai R, Tomita H, Hirose $\mathrm{Y}$, et al. Augmented therapeutic efficacy of an oncolytic herpes simplex virus type 1 mutant expressing ICP34.5 under the transcriptional control of musashi1 promoter in the treatment of malignant glioma. Hum Gene Ther 2007; 18: 63-73.

[35] Debinski W, Tatter SB. Convection-enhanced delivery for the treatment of brain tumors. Expert Rev Neurother 2009; 9: 1519-27.

[36] Hadjipanayis CG, Fellows-Mayle W, Deluca NA. Therapeutic efficacy of a herpes simplex virus with radiation or temozolomide for intracranial glioblastoma after convection-enhanced delivery. Mol Ther 2008; 16: 1783-8.

[37] Papanastassiou V, Rampling R, Fraser M, et al. The potential for efficacy of the modified (ICP 34.5(-)) herpes simplex virus HSV1716 following intratumoural injection into human malignant glioma: a proof of principle study. Gene Ther 2002; 9: 398-406.

[38] Harrow, S, Papanastassiou V, Harland J, et al. HSV1716 injection into the brain adjacent to tumour following surgical resection of high-grade glioma: safety data and long-term survival. Gene Ther 2004; 11: 1648-58.

[39] Shah AC, Benos D, Gillespie GY, Markert JM. Oncolytic viruses: clinical applications as vectors for the treatment of malignant gliomas. J Neurooncol 2003; 65: 203-26.

(C) Cassady and Parker; Licensee Bentham Open.

This is an open access article licensed under the terms of the Creative Commons Attribution Non-Commercial License (http: //creativecommons.org/licenses/by-nc/ 3.0/) which permits unrestricted, non-commercial use, distribution and reproduction in any medium, provided the work is properly cited. 